\title{
Stretchable gas barrier films achieved by hydrogen-bond self-assembly of nano-brick multilayers
}

\author{
Kaiqiang $\mathrm{Shi}^{1}$, Xiaozhi $\mathrm{Xu}^{1}$, Siyuan Dong ${ }^{1}$, Biao $\mathrm{Li}^{1}$, and Jingbin $\mathrm{Han}^{1}$ \\ ${ }^{1}$ Beijing University of Chemical Technology
}

January 29, 2021

\begin{abstract}
Stretchable gas barrier films achieved by hydrogen-bond self-assembly of nano-brick multilayers
\end{abstract}

Stretchable gas barrier films achieved by hydrogen-bond self-assembly of nano-brick multilayers

Kaiqiang Shi, Xiaozhi Xu, Siyuan Dong, Biao Li and Jingbin Han*

State Key Laboratory of Chemical Resource Engineering, Beijing Advanced Innovation Center for Soft Matter Science and Engineering, Beijing University of Chemical Technology, Beijing 100029, P. R. China

\section{Author Information}

* Corresponding authors. Phone: +86-10-64412131. Fax: +86-10-64425385. E-mail: hanjb@mail.buct.edu.cn.

Abstract: Stretchable gas barrier films hold great application potentials in flexible electronics, sensors and food/drug packaging. Inorganic-organic hybrid films containing two-dimensional nanosheets have shown good gas barrier performance, but moderate tensile property, because of the rigid characteristics of covalent or ionic bonds between the assembly units. In this work, we used LDH nanosheets rich in hydroxyl groups as building units, followed by modification of tannic acid (TA), to assemble with polyethylene oxide (PEO) through hydrogen bonds. Compared with previous work, the tensile property and oxygen barrier performance of $(\mathrm{TA} @ \mathrm{LDH} / \mathrm{PEO})_{n}$ films have been significantly improved. A fifty-bilayer TA@LDH/PEO film (865 nm thick), deposited on a $1 \mathrm{~mm}$ thick natural rubber substrate, results in a $29 \times$ reduction (contrast with bare substrate) in oxygen transmission rate and maintains its good barrier property even under a large elongation of $120 \%$. The excellent tensile and gas barrier properties are attributed to the ductility of hydrogen bond network among building blocks and the significant prolongation of oxygen transmission path induced by LDH nanosheets. This work provides a simple and cost-effective strategy for the preparation of nanosheetsbased hybrid materials, which are useful in those fields requiring simultaneously high gas barrier and good flexibility.

KEYWORDS: films, membrane materials, two-dimensional nanosheets, gas barrier,

hydrogen bonds assembly

\section{Introduction}

Gas barrier films have a wide range of applications in food/drug preservation and electronic device encapsulation. ${ }^{1-5}$ The protection of flexible displays, inflation/deflation of tires and football/basketball require deformation. So the demand for flexible and stretchable gas barrier films has greatly increased. Conventional 
alumina/zinc oxide coatings display excellent barrier properties but are easy to break or crack when the barrier films are stretched. Although pure polymer materials such as ethylene/vinyl alcohol copolymer (EVOH), polyvinyl alcohol (PVA), and polyvinylidene chloride (PVDC) can meet the requirement of general packaging application, they cannot be applied to high barrier fields. ${ }^{6-12}$ Besides, such polymer materials have limited application range due to their humidity sensitivity and poor mechanical strength. By hybridizing inorganic nanoplatelets (such as hydrotalcite and montmorillonite) with polymers, multilayer organic-inorganic films called "nano-brick" solve the problem of poor gas barrier property of polymers and simultaneously improve the mechanical property. ${ }^{13-18}$

Layer-by-layer (LBL) assembly is an effective method to prepare gas barrier coatings through nanoscale control of film architecture. ${ }^{19-23}$ Electrostatic layer assembly technology is the earliest and most common method for constructing multilayer films. ${ }^{13,} 24$ This method is suitable for assembly between oppositely charged elements, such as polymer electrolytes, inorganic nanoparticles, two-dimensional (2D) nanoplatelets, etc. Although the electrostatically assembled films have good barrier properties, these nano-walls are usually very strong and cracks upon stretching. Stretchable gas barrier films must be crack-free because the cracks act as gas transmission highways. For example, a $10 \%$ strain resulted in cracking of PEI/montmorillonite nanocoating and thus an increase in gas permeability by an order of magnitude. ${ }^{25,}{ }^{26}$ Hydrogen bonded multilayer films are generally less rigid than ionically bonded films. ${ }^{27-29}$ The weaker hydrogen bonds lead to a looser film structure with a lighter crosslink density that produces greater strain without damage. However, extensibility generally increases at the expense of gas permeability because the barrier properties of LBL deposited films are dependent on density and density is related to hardness. One strategy to break through this limitation is to modify the hydrogen bonding system to increase barrier properties while maintaining its extensibility.

Hydrotalcite, referred to as layered double hydroxide (LDH), is an important 2D layered material. ${ }^{30-34}$ The surface of the LDH laminate contains a large number of hydroxyl groups, and the presence of trivalent metal ions in metal-oxygen hexahedra makes the laminate positively charged. Therefore, LDH can be incorporated into hydroxyl group-containing or negatively-charged polymer matrixvia hydrogen bond or electrostatic interaction, respectively. In our recent work, we used LDH nanoplatelets to construct high-barrier film materials through electrostatic force ${ }^{5}$ However, the barrier properties of such materials will decrease during long-term use or under bending conditions, which restricts their application in the fields that require good flexibility and stretchability.

In this work, we prepared single-layer LDH nanosheets followed by surface modification with tannic acid (TA) (named as TA@LDH), which were alternately assembled with polyethylene oxide (PEO) to prepare hybrid films with stretchable gas barrier property. It is revealed that the combination of TA@LDH into PEO prolongs the diffusion path of the gas molecules, resulting in enhanced gas barrier properties. The ability to prevent cracking and preserve the gas barrier up to $120 \%$ elongation provides a tremendous opportunity for improving the barrier of elastomeric materials. After several times of stretching $100 \%$, the film still has a high oxygen barrier property. The hydrogen bond results in more loose film structure, lighter crosslinking density, and larger strain without damage. Due to the abundant hydrogen bond sites on the LDH surface, stretching may lead to the transfer of hydrogen bond from one bonding site to another, rather than fracture. These highly elastomeric assemblies are potentially useful for light-weighting inflatable devices, tyres and wrapping.

\section{Experimental section}

2.1 Reagents and Materials. Analytically pure $\mathrm{Mg}\left(\mathrm{NO}_{3}\right)_{2} * 6 \mathrm{H}_{2} \mathrm{O}, \mathrm{Al}\left(\mathrm{NO}_{3}\right)_{3} * 9 \mathrm{H}_{2} \mathrm{O}$ were purchased from Beijing Chemical Plants. Urea and ammonia were obtained from Xilong Scientific Co., Ltd. TA was purchased from Aladdin Biotechnology Co., Ltd. PEO was obtained from Maclean Biochemical Technology Co., Ltd. Natural rubber (NR) substrate was purchased from ASONE (Shanghai) Trading Co., Ltd. All the reagents were used without further purification. Deionized (DI) and decarbonated water was used in all the experiments. 
2.2 Preparation of colloidal MgAl-LDH nanosheets suspension. $\mathrm{MgAl}\left(\mathrm{CO}_{3}\right)-\mathrm{LDH}$ was prepared by urea-assisted hydrothermal method. ${ }^{14}$ Specifically, $25.64 \mathrm{~g}$ of $\mathrm{Mg}\left(\mathrm{NO}_{3}\right)_{2} * 6 \mathrm{H}_{2} \mathrm{O}, 18.70 \mathrm{~g}$ of $\mathrm{Al}\left(\mathrm{NO}_{3}\right)_{3} * 9 \mathrm{H}_{2} \mathrm{O}$, and $30.03 \mathrm{~g}$ of urea were dissolved in $500 \mathrm{ml}$ of water and placed in a stainless steel autoclave lined with polytetrafluoroethylene. After hydrothermal treatment at $110 \mathrm{degC}$ for $24 \mathrm{~h}$, it was then washed several times with a centrifuge using deionized water and ethanol. After cooling, the precipitate of $\operatorname{MgAl}\left(\mathrm{CO}_{3}\right)-\mathrm{LDH}$ nanoplatelets was washed several times with deionized water and ethanol and dried at room temperature.

Then the interlayered $\mathrm{CO}_{3}{ }^{2-}$ were exchanged by $\mathrm{NO}_{3}{ }^{-}$ions through the salt-acid treatment of $\mathrm{MgAl}\left(\mathrm{CO}_{3}\right)$ $\mathrm{LDH}$, to facilitate delamination. ${ }^{35}$ The sample of $1 \mathrm{~g} \mathrm{MgAl}\left(\mathrm{CO}_{3}\right)-\mathrm{LDH}$ nanoplatelets was added into $1 \mathrm{~L}$ aqueous salt-acid solution $\left(311 \mu \mathrm{L}\right.$ of $\mathrm{HNO}_{3}, 98 \mathrm{wt} \%$ and $127 \mathrm{~g}$ of $\left.\mathrm{NaNO}_{3}\right)$ and stirred for $24 \mathrm{~h}$ in a nitrogen atmosphere. The $\mathrm{MgAl}\left(\mathrm{NO}_{3}\right)-\mathrm{LDH}$ nanoplatelets were collected by centrifugation and washed with water and ethanol and dried in a vacuum oven. Afterward, samples of $0.1 \mathrm{~g} \operatorname{MgAl}\left(\mathrm{NO}_{3}\right)-\mathrm{LDH}$ nanoplatelets were placed into $100 \mathrm{~mL}$ formamide for $48 \mathrm{~h}$ with stirring to prepare single-layer nanosheets suspension. ${ }^{36}$

2.3 Preparation of TA@LDH suspension. TA was put into formamide and stirred at room temperature until complete dissolution. Under the protection of $\mathrm{N}_{2}, \mathrm{LDH}$ nanosheet suspension was mixed with formamide solution of TA and stirred at room temperature for 24 hours. TA@LDH suspension can be obtained through the above operations. To study the effect of TA: LDH ratio on the gas resistance and tensile properties of the films, different TA@LDH suspensions with various TA: LDH ratios were prepared. To ensure that the total concentration of TA@LDH suspension is $0.1 \mathrm{wt} \%$ and $\mathrm{pH}$ is 8.0, four groups of experiments were carried out according to TA: $\mathrm{LDH}=1: 1,2: 1,3: 1$ and 4:1. Normally, the expression of TA@LDH represents the sample with TA: $\mathrm{LDH}=3: 1$, unless specified.

2.4 Fabrication of (TA@LDH/PEO $)_{n}$ multilayer films. An aqueous solution of PEO (0.1 wt\%) was stirred at $60{ }^{\circ} \mathrm{C}$ for $2 \mathrm{~h}$ to achieve homogeneity. The $\mathrm{pH}$ of each PEO solution and rinsing DI water was altered to 8.0 using ammonia. Multilayer films of $(\mathrm{TA} @ \mathrm{LDH} / \mathrm{PEO})_{n}$ were fabricated by applying the LBL assembly procedure. Quartz glass, silicon wafer and NR film (thickness: $\sim 1 \mathrm{~mm}$ ) were chosen as substrates for various characterizations and tests. Quartz glass substrate was used for UV-vis absorption spectra characterization, and silicon wafer was applied for SEM and AFM study. All the other measurements were based on the samples on NR substrate. Before assembly, every NR film was rinsed with DI water, soaked in n-propanol at $40{ }^{\circ} \mathrm{C}$ for $10 \mathrm{~min}$, then treated with n-propanol and deionized water by ultrasound at $40{ }^{\circ} \mathrm{C}$, and dried naturally. Quartz glass and silicon wafer were firstly washed in $30 \% \mathrm{H}_{2} \mathrm{O}_{2} / \mathrm{NH}_{3} \cdot \mathrm{H}_{2} \mathrm{O}(\mathrm{v} / \mathrm{v}=3: 7)$ and concentrated $\mathrm{H}_{2} \mathrm{SO}_{4}$ for 30 min each to make the substrate surface hydrophilic and negatively charged. The overall LBL process consists of a cyclic repetition of the following steps: (a) dipping the pre-treated substrate into a colloidal TA@LDH suspension for $10 \mathrm{~min}$, followed by rinsing with DI water thoroughly; (b) immersing into an aqueous solution of PEO for 10 min and washed with DI water. Multilayer films of (TA@LDH/PEO) ${ }_{n}$ were fabricated by alternate deposition of TA@LDH suspension and PEO solution for $n$ cycles. The resulting films were finally rinsed with water and dried at ambient temperature. As for comparison samples, the (TA/LDH $)_{n}$ , $(\mathrm{TA} / \mathrm{PEO})_{n}$ and $(\mathrm{LDH} / \mathrm{PEO})_{n}$ films were prepared by a similar assembly procedure in a sequence of $\mathrm{LDH}$ nanosheets suspension, TA, PEO aqueous solution. All films were prepared using an automatic dip-coating machine (Figure S1, Supporting Information). A stretching instrument (Figure S2, Supporting Information) was used to apply different strains to the films with and without nanocoatings for $1 \mathrm{~min}$.

\section{Results and discussion}

XRD pattern of the $\operatorname{MgAl}\left(\mathrm{CO}_{3}\right)-\mathrm{LDH}$ sample (Figure 1A) shows clear diffraction peaks at $12.2^{\circ}, 24.3^{\circ}$, $34.9^{\circ}, 39.1^{\circ}, 47.2^{\circ}, 61.2^{\circ}$ and $62.3^{\circ}$, which correspond to (003), (006), (009), (015), (018), (110) and (113) diffractions of a hydrotalcite phase. ${ }^{37}$ To delaminate $\mathrm{LDH}$ into monolayer, the interlayered $\mathrm{CO}_{3}{ }^{2-}$ were replaced by $\mathrm{NO}_{3}{ }^{-}$ions. Figure 1B displays the (003) characteristic peak shifts from 12.2deg to $10.2 \mathrm{deg}$ and the corresponding layer spacing expands from $0.77 \mathrm{~nm}$ to $0.89 \mathrm{~nm}$, which confirms that the anions between the hydrotalcite layers have been exchanged from carbonate to nitrate. Figure 1C and 1D are the scanning electron microscopy (SEM) images of $\mathrm{MgAl}\left(\mathrm{CO}_{3}\right)-\mathrm{LDH}$ and $\mathrm{MgAl}\left(\mathrm{NO}_{3}\right)-\mathrm{LDH}$. It is found that they have similar regular hexagonal shape, and their particle size distribution is around 1.7-2.5 $\mu \mathrm{m}$. 

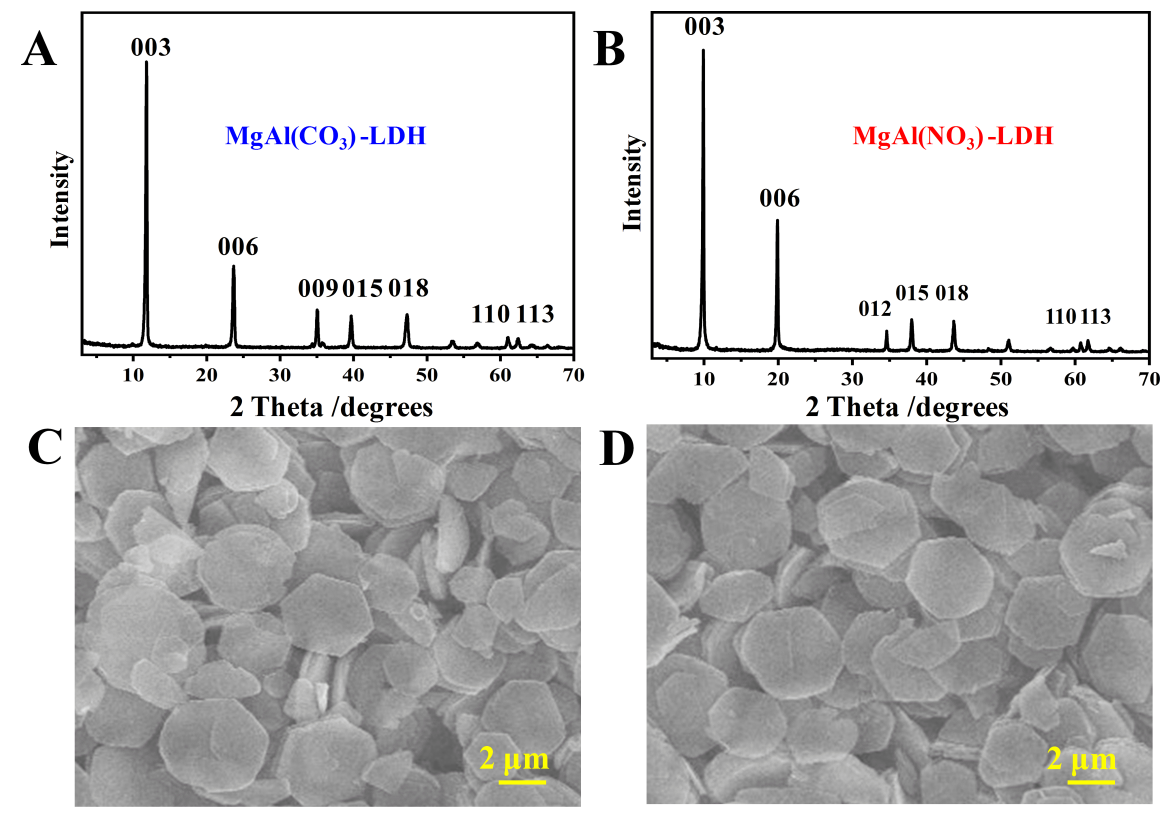

Figure 1. XRD patterns and SEM images of $(\mathrm{A}) /(\mathrm{C}) \mathrm{MgAl}\left(\mathrm{CO}_{3}\right)-\mathrm{LDH}$ and (B)/(D) $\mathrm{MgAl}\left(\mathrm{NO}_{3}\right)-\mathrm{LDH}$.

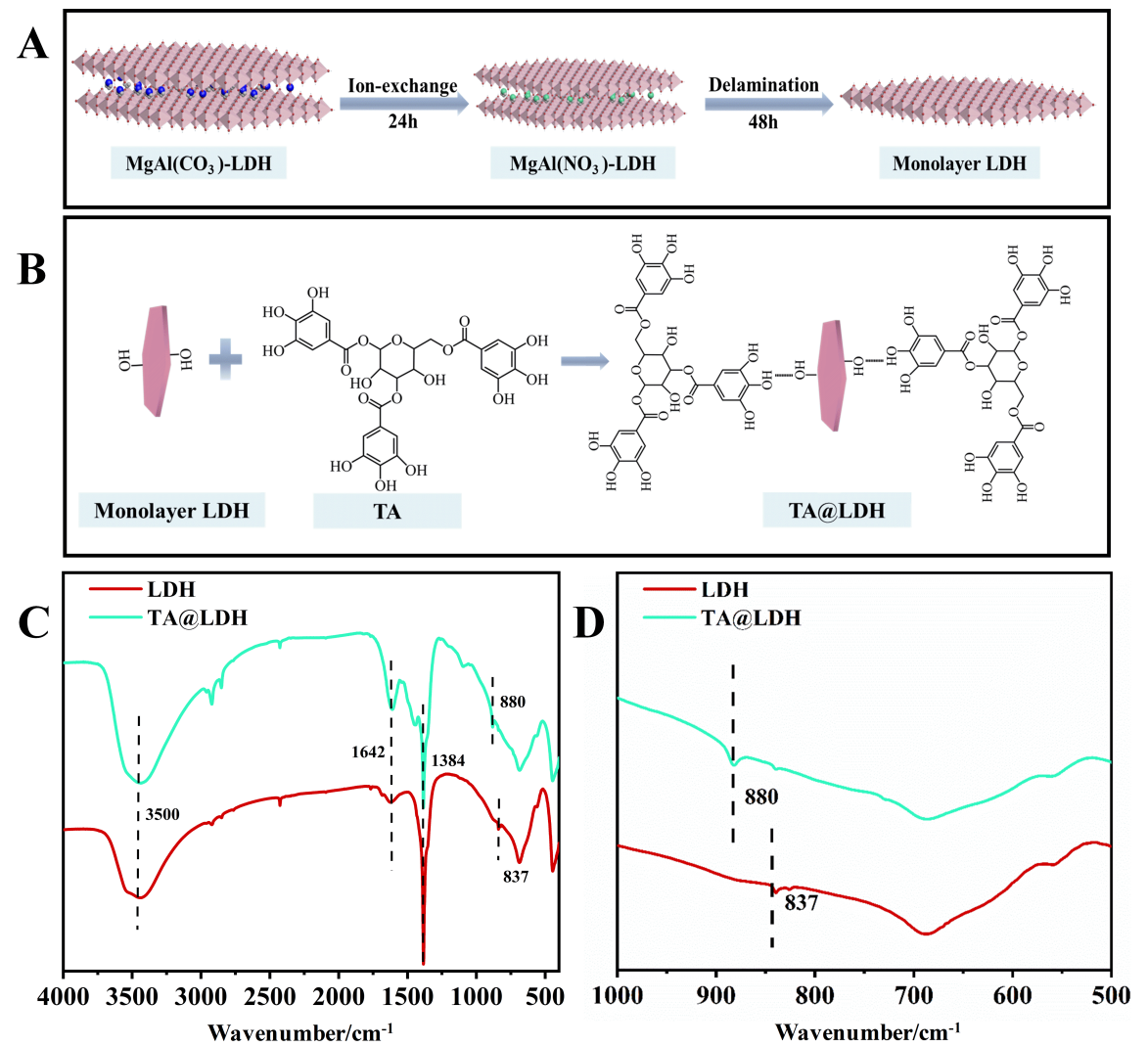

Figure 2. (A) Schematic diagram of preparation process of monolayer LDH nanosheets; (B) Schematic diagram of LDH modification by TA; (C) FTIR spectra of LDH and TA@LDH; (D) Partial magnification of 
FTIR spectra.

Single-layer LDH nanosheets rich in hydroxyl groups were obtained by delaminating $\mathrm{MgAl}\left(\mathrm{NO}_{3}\right)-\mathrm{LDH}$ in formamide (Figure 2A). Then, the surface of the monolayer LDH nanosheets was modified by TA (Figure 2B). Upon irradiation by a red laser, the LDH and TA@LDH suspensions both show clear Tyndall effect, indicating their homogeneity (Figure S3 A and B, Supporting information). The interaction between TA and LDH was investigated by FTIR spectra (Figure 2C). The LDH and TA@LDH both display obvious absorption peaks at $3500 \mathrm{~cm}^{-1}$ and $1642 \mathrm{~cm}^{-1}$, which are ascribed to stretching and bending vibration absorption of O-H. For LDH sample, a strong sharp absorption peak at $1384 \mathrm{~cm}^{-1}$ is attributed to the antisymmetric stretching vibration of $\mathrm{NO}_{3}{ }^{-}$. Bending vibration of M-O-M appears at $837 \mathrm{~cm}^{-1}$, which shifts to $880 \mathrm{~cm}^{-1}$ after hybridization with TA. The blue shift of M-O-M absorption band for the TA@LDH sample suggests the formation of hydrogen bonds between TA and LDH.
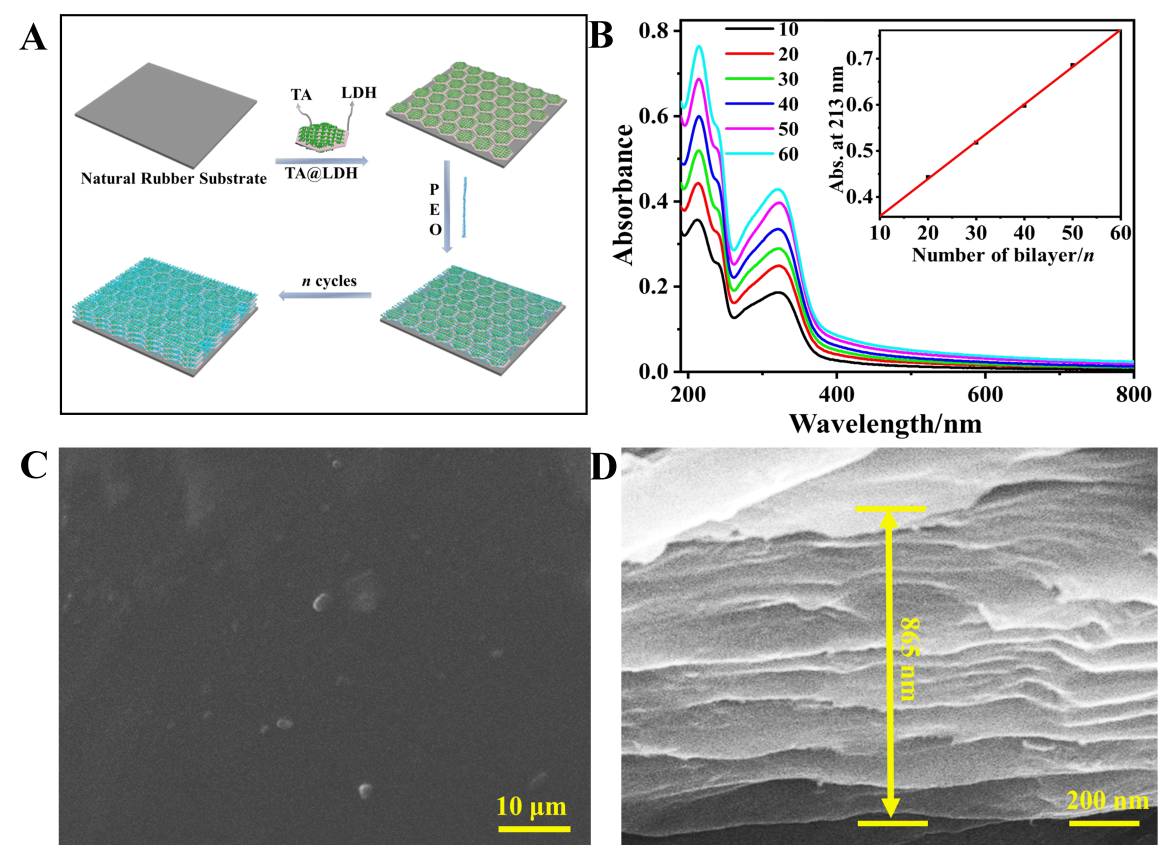

Figure 3. (A) Schematic illustration for the assembly of (TA@LDH/PEO) ${ }_{n}$ films by LBL method; (B) UV-vis absorption spectra of the (TA@LDH/PEO $)_{n}(n=10-70)$ films on quartz glass substrates (inset: the linear relationship between the absorbance at $213 \mathrm{~nm}$ and the bilayer number $n$ ); (C) Top-view and (D) cross-sectional SEM images of a (TA@LDH/PEO) $)_{50}$ film.

$(\mathrm{TA} @ \mathrm{LDH} / \mathrm{PEO})_{n}$ multilayer films were fabricated through layer-by-layer technique on pre-treated substrates (Figure 3A). UV-vis spectra (Figure 3B) of the as-prepared (TA@LDH/PEO) ${ }_{n}$ films show two intense absorption bands at $213 \mathrm{~nm}$ and $338 \mathrm{~nm}$, attributed to the characteristic absorption of TA. The absorbance increases linearly (Figure 3B, inset) relative to the number of deposition cycles, illustrating the well-proportioned and gradual growth of the multilayer films. According to the molecular structure of PEO (Figure S3 C, Supporting Information) and TA (Figure 2B), hydrogen bond network can be formed in the $(\mathrm{TA} @ \mathrm{LDH} / \mathrm{PEO})_{n}$ films.

Top-view SEM image of a (TA@LDH/PEO) 50 film with a continuous and smooth surface is shown in Figure 3C. Clay alignment in the multilayer films is clearly observed using cross-sectional SEM (Figure 3D) image of 50 bilayer film deposited on silicon wafer with a thickness of $865 \mathrm{~nm}$. The image shows a clear layered structure with LDH nanosheets shown in white lines, which illustrates good dispersity and high orientation of the LDH nanosheets. The 2D nanosheets with a perfect parallel orientation to the substrate will play the 
best role in the gas barrier function.

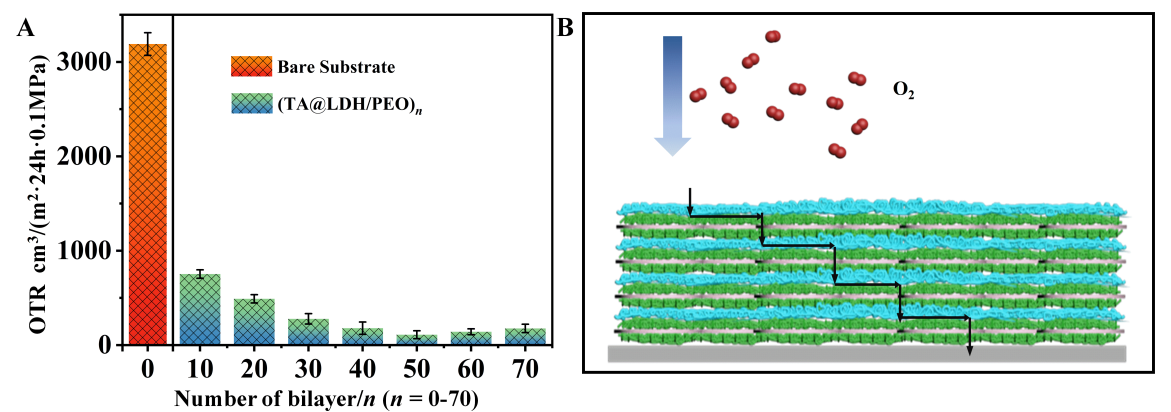

Figure 4. (A) OTR of (TA@LDH/PEO $)_{n}(n=10-70)$ films with different bilayer numbers; (B) Schematic diagram of gas penetration in a barrier film.

The bare NR substrate displays an oxygen transmission rate (OTR) of $3190 \mathrm{~cm}^{3} /\left(\mathrm{m}^{2} \cdot 24 \mathrm{~h} \cdot 0.1 \mathrm{MPa}\right)$, as shown in Figure 4A. Upon deposition of (TA@LDH/PEO) ${ }_{n}$ coatings, the OTR values undergo a significant decrease. With an increase in the number of the assembly layers, the permeability decreases because of the barrier effect caused by horizontal LDH nanosheets. The improved oxygen barrier properties are attributed to the good 2D ordering of LDH nanosheets, which increases the diffusion path of oxygen molecules, commonly known as tortuous pathway (Figure 4B). The best oxygen resistance performance can be obtained when the composite film has 50 layers $\left(\mathrm{OTR}=110 \mathrm{~cm}^{3} /\left(\mathrm{m}^{2} \cdot 24 \mathrm{~h} \cdot 0.1 \mathrm{MPa}\right)\right)$. When the number of $(\mathrm{TA} @ \mathrm{LDH} / \mathrm{PEO})_{n}$ film layers continues to increase, the oxygen barrier performance begins to decline. This is probably because when there are too many layers and the film is too thick, the substrate will be hard to bear so that the composite film will fall out or crack. Therefore, 50 layers are selected as the best number of bilayers.

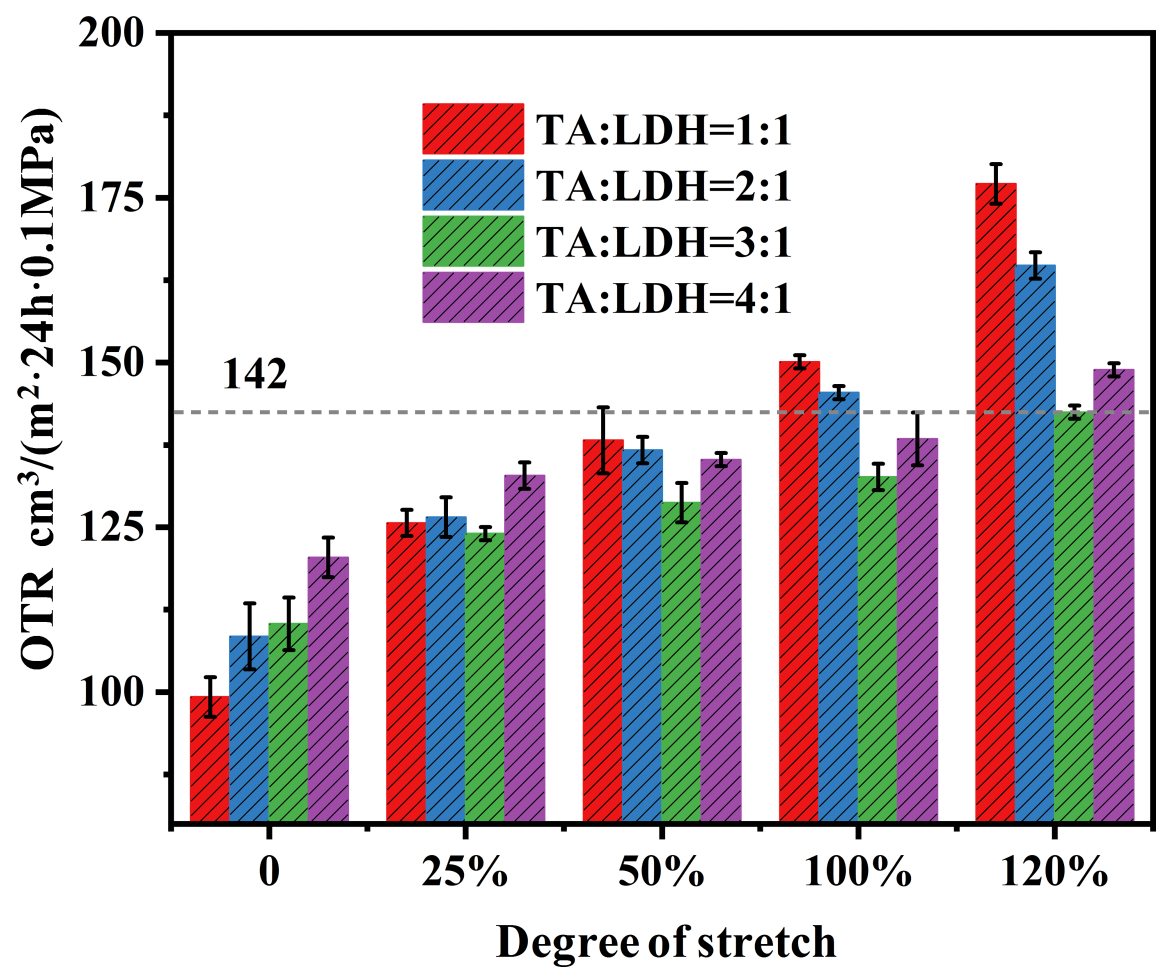


Figure 5. OTR of (TA@LDH/PEO) 50 hybrid films with different ratios of TA: LDH.

Hydrogen bonds are much weaker than electrostatic interactions but stronger than van der Waals forces. Therefore, hydrogen-bonded films have good tensile properties. ${ }^{27}$ Because hydrogen bonding is very important for mechanical properties, we regulated the ratio of TA to LDH. The OTR of four kinds of thin films with different ratios and layers were measured. Besides the (TA@LDH/PEO) 50 film with TA: LDH of 3: 1, the samples with other proportions (1:1,2:1 and 4:1) also exhibit the same change trend for OTR with increasing the bilayer number (Figure S4, Support Information).

No matter what the ratio of TA to $\mathrm{LDH}$, the OTR of the films increased slightly after stretching (Figure 5). The oxygen resistance of the film is still good after stretching. However, with the decrease of LDH content in TA@LDH, the attenuation of the oxygen barrier is slower upon stretching. When the ratio of LDH nanosheets is $50 \%$, the OTR of $120 \%$ stretching is $79 \%$ higher than that without stretching (from 99 to $\left.177 \mathrm{~cm}^{3} /\left(\mathrm{m}^{2} \cdot 24 \mathrm{~h} \cdot 0.1 \mathrm{MPa}\right)\right)$. When the ratio of $\mathrm{LDH}$ is $20 \%$, the OTR of $120 \%$ stretching is only $23 \%$ higher than that without stretching (from 120 to $149 \mathrm{~cm}^{3} /\left(\mathrm{m}^{2} \cdot 24 \mathrm{~h} \cdot 0.1 \mathrm{MPa}\right.$ )). These results show a higher content of TA is favorable to the film ductility due to the presence of elastic hydrogen network, although the incorporation of LDH nanosheet improves the barrier property.

Considering the balance between oxygen barrier and tensile properties, the best ratio of LDH is $25 \%$ (i.e. TA: $\mathrm{LDH}=3: 1)$. At 50 -bilayer, the OTR is $110 \mathrm{~cm}^{3} /\left(\mathrm{m}^{2} \cdot 24 \mathrm{~h} \cdot 0.1 \mathrm{MPa}\right)$, and its oxygen barrier capacity is $28 \times$ higher than that of NR substrate. When the stretch ratio was $25 \%$, the OTR increased by $12 \%$ to 124 $\mathrm{cm}^{3} /\left(\mathrm{m}^{2} \cdot 24 \mathrm{~h} \cdot 0.1 \mathrm{MPa}\right)$. The OTR is $128 \mathrm{~cm}^{3} /\left(\mathrm{m}^{2} \cdot 24 \mathrm{~h} \cdot 0.1 \mathrm{MPa}\right)$ at $50 \%$ stretching, which is $17 \%$ higher than that of the unstrained film. It still has good oxygen resistance at $100 \%$ stretching, with OTR increased by $20 \%$ to $133 \mathrm{~cm}^{3} /\left(\mathrm{m}^{2} \cdot 24 \mathrm{~h} \cdot 0.1 \mathrm{MPa}\right)$.

As shown in Table 1, the (TA@LDH/PEO) 50 hybrid film has significant advantages over other films in published work. Although the oxygen barrier property of (TA@LDH/PEO) $)_{50}$ film is not the best, its tensile deformation ratio reaches $120 \%$, which is not achieved by other works. And after $120 \%$ stretching, the permeability is only $29 \%$ higher than that without stretching. It can be found that increasing the tensile capacity needs to sacrifice part of the oxygen barrier capacity. Even so, our (TA@LDH/PEO) $)_{50}$ film shows simultaneously excellent tensile property and competitive oxygen barrier capability.

Table 1. Comparative study on gas barrier performance after stretching between our (TA@LDH/PEO) 50 film and reported materials

\begin{tabular}{llll}
\hline Film Materials & Tensile Deformation & Permeability Reduce (relative to the blank substrate) & Permeability Inc \\
\hline${\text { (TA@LDH/PEO })_{50}}_{(\mathrm{PAA} / \mathrm{PEO})_{20}}$ & $120 \%$ & $29 \times$ & $29 \%$ \\
$(\mathrm{PEI} / \mathrm{PAA} / \mathrm{PEO} / \mathrm{PAA})_{20}$ & $100 \%$ & $10 \times$ & $70 \%$ \\
$(\mathrm{PPM} / \mathrm{PEO})_{20}$ & $20 \%$ & $15 \times$ & $138 \%$ \\
$(\mathrm{C}-\mathrm{PU} / \mathrm{GO} / \mathrm{A}-\mathrm{PU})_{40}$ & $15 \%$ & $80 \times$ & $20 \%$ \\
\hline
\end{tabular}



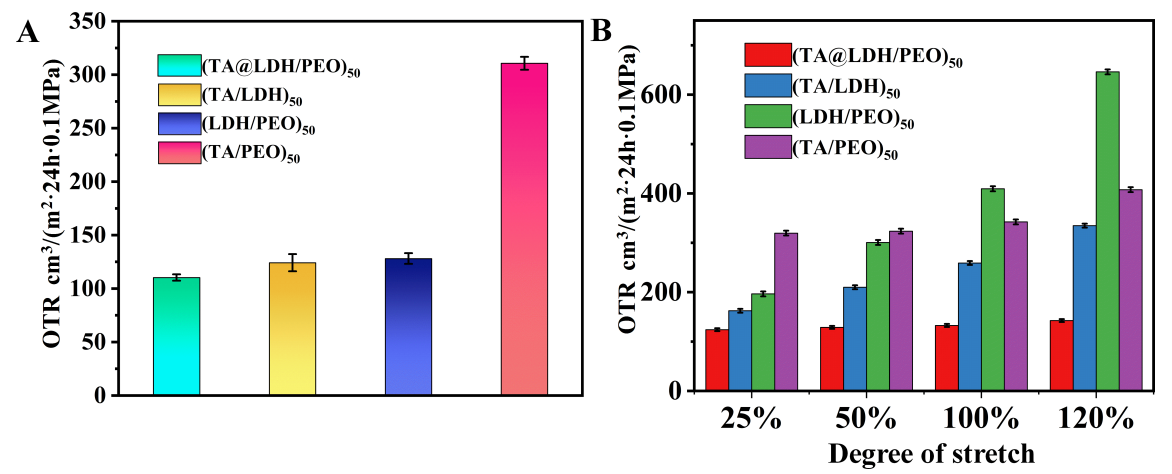

Figure 6. (A) OTR of four different composite films: (TA@LDH/PEO $)_{50},(\mathrm{TA} / \mathrm{LDH})_{50},(\mathrm{LDH} / \mathrm{PEO})_{50}$ and $(\mathrm{TA} / \mathrm{PEO})_{50} ;(\mathrm{B})$ OTR of these composite films after stretching.

Three films $(\mathrm{TA} / \mathrm{LDH})_{50},(\mathrm{LDH} / \mathrm{PEO})_{50}$ and $(\mathrm{TA} / \mathrm{PEO})_{50}$ were prepared as control samples and compared with $(\mathrm{TA} @ \mathrm{LDH} / \mathrm{PEO})_{50}$. As shown in Figure $6 \mathrm{~A}$, we can find that the (TA/PEO) $)_{50}$ composite film shows the worst oxygen barrier ability, which is only $10 \times$ lower than that of NR substrate at 50-bilayer $(\mathrm{OTR}=$ $\left.311 \mathrm{~cm}^{3} /\left(\mathrm{m}^{2} \cdot 24 \mathrm{~h} \cdot 0.1 \mathrm{MPa}\right)\right)$. The reason why $(\mathrm{TA} / \mathrm{PEO})_{50}$ composite films have poor oxygen barrier ability than other films is due to the absence of LDH. (TA@LDH/PEO) ${ }_{50}$ composite films have the best properties, which might be because $(\mathrm{TA} @ \mathrm{LDH} / \mathrm{PEO})_{50}$ has better interfacial compatibility and richer hydrogen bond networks than $(\mathrm{TA} / \mathrm{LDH})_{50}\left(\mathrm{OTR}=124 \mathrm{~cm}^{3} /\left(\mathrm{m}^{2} \cdot 24 \mathrm{~h} \cdot 0.1 \mathrm{MPa}\right)\right)$ and $(\mathrm{LDH} / \mathrm{PEO})_{50}(\mathrm{OTR}=128$ $\left.\mathrm{cm}^{3} /\left(\mathrm{m}^{2} \cdot 24 \mathrm{~h} \cdot 0.1 \mathrm{MPa}\right)\right)$. Hydrogen bonded films can increase the diffusion residence of oxygen molecules and enhance the barrier ability of the films. The OTR of other films with various assembly numbers also shows that (TA@LDH/PEO) ${ }_{n}$ has the best performance (Figure S5, Support Information).

The morphology of these samples under different tensile conditions was studied. When the stretch ratio is $25 \%$, cracks appear in the $(\mathrm{LDH} / \mathrm{PEO})_{50}$ film (Figure 7B), and the OTR increases by $53 \%$ (from 128 to $\left.196 \mathrm{~cm}^{3} /\left(\mathrm{m}^{2} \cdot 24 \mathrm{~h} \cdot 0.1 \mathrm{MPa}\right)\right)$. At $120 \%$ stretching, OTR increased by $404 \%$. Similar to $(\mathrm{LDH} / \mathrm{PEO})_{50}$ film, the $(\mathrm{TA} / \mathrm{LDH})_{50}$ film also exhibits a disappointing oxygen barrier after stretching. However, the stretch ratio of $(\mathrm{TA} / \mathrm{LDH})_{50}$ film is better than that of $(\mathrm{LDH} / \mathrm{PEO})_{50}$. Cracks appeared at $50 \%$ tension (Figure $7 \mathrm{E})$ and OTR reached $210.03 \mathrm{~cm}^{3} /\left(\mathrm{m}^{2} \cdot 24 \mathrm{~h} \cdot 0.1 \mathrm{MPa}\right)$. Although the oxygen resistance of $(\mathrm{TA} / \mathrm{PEO})_{50}$ thin films is poor, its tensile properties are excellent. Only when the stretch ratio reaches $120 \%$ (Figure 6J), the small cracks appear. At this time, its OTR increased by $31 \%$. TA effectively acts as a small molecule cross-linker between flexible PEO chains, posing very little restriction to chain segment mobility within the TA/PEO assembly. The non-rigid hydrogen bonding network between TA and PEO provides excellent tensile properties. Similarly, the (TA@LDH/PEO) $)_{50}$ film also has small cracks when stretched by $120 \%$. The oxygen barrier ability of $(\mathrm{TA} @ \mathrm{LDH} / \mathrm{PEO})_{50}$ film is much higher than that of $(\mathrm{TA} / \mathrm{PEO})_{50}$ film, while these two films show similar stretchability. The excellent properties of (TA@LDH/PEO) $)_{50}$ films are attributed to the bridging effect of TA at the interface of $\mathrm{LDH}$ and $\mathrm{PEO}$, which provide stretchable elastic network to tolerate the external force. 


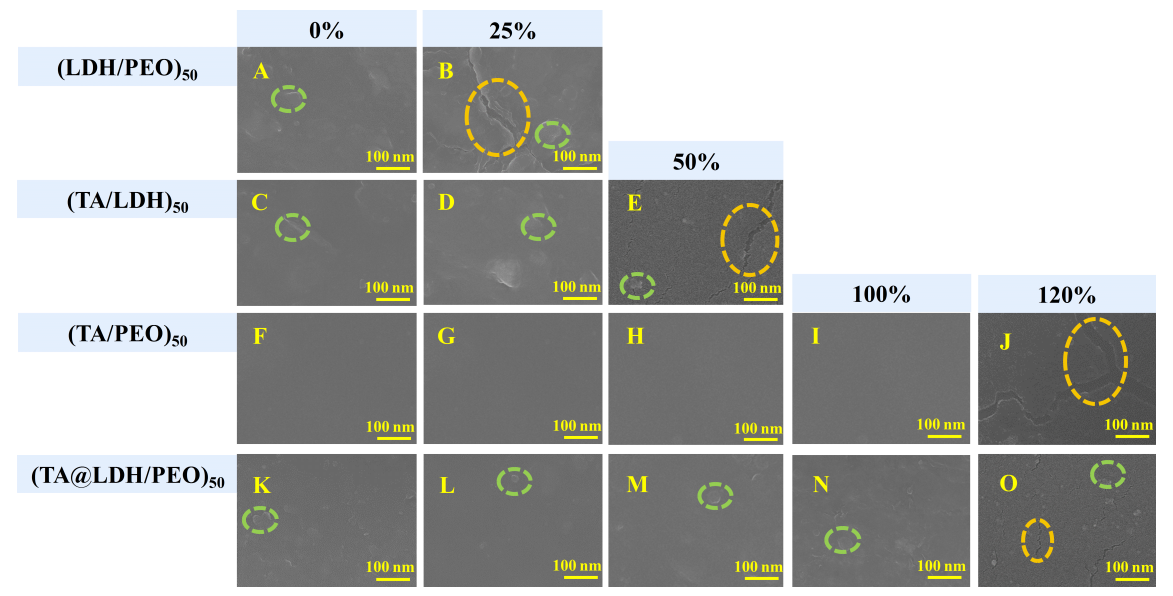

Figure 7. SEM images of four kinds of composite films after stretching. (Green circles mark the aggregation of LDH nanosheets and orange circles represent the fracture of the film.)

The cyclic stability under tensile condition was further investigated. Because of the generation of small cracks in $120 \%$ deformation, the repeated tensile test was carried out under $100 \%$ deformation. It can be found that the OTR of (TA@LDH/PEO) 50 films does not change much after stretching (Figure S6, Support Information). With the increase of the recycle times, the gas blocking performance only slowly decays, and after 10 recycle times, it decreases by only $20 \%$. The excellent performance after multiple stretching makes the $(\mathrm{TA} @ \mathrm{LDH} / \mathrm{PEO})_{50}$ film a promising candidate for stretchable gas barrier material.

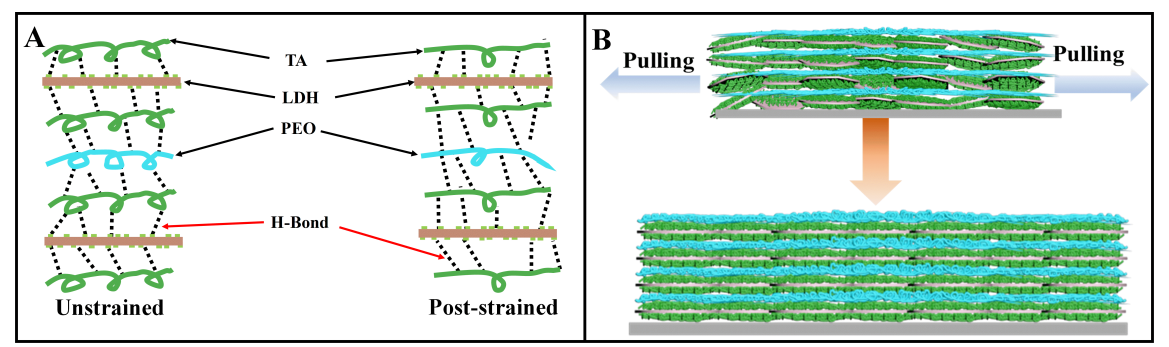

Figure 8. (A) Schematic diagram of the hydrogen bond networks in the (TA@LDH/PEO) ${ }_{n}$ films; (B) Reorientation of LDH nanosheets to perfect parallel alignment after stretching.

Whether upon repeated stretching or at a high stretching ratio, the (TA@LDH/PEO) $)_{50}$ film keeps very good oxygen barrier performance. Many factors make it have good oxygen resistance and stretchable ability. LDH, TA and PEO all have plentiful hydrogen bonding sites. When the film deformation is small, the Hbonding between TA@LDH and PEO has much higher bond strength than van der Waals forces between PEO chains, so the deformation is more likely to be a result of PEO chain segment reorientation and extension rather than breaking hydrogen bonds. In the process of further stretching, hydrogen bond damage and regeneration of the film occur continuously (Figure 8A). In addition, some LDH nanosheets that are not strictly parallel to the substrate will reorient after stretching to improve the oxygen barrier capability of the film (Figure 8B). Therefore, the (TA@LDH/PEO) 50 film simultaneously shows good barrier property and excellent stretchability.

\section{Conclusions}

This work uses layers of self-assembly (based on hydrogen bonding) to prepare stretchable gas barrier films. The combination of a high stretch ratio is the most distinct feature in this work compared with other 
inorganic platelet-reinforced polymers as gas barrier films. The LDH nanosheets as well as the hydrogen bonding network in this work induce a long diffusion length, thus suppressing the oxygen permeability. Based on the extensibility of hydrogen bond, the composite film has good oxygen resistance after largedeformation and repeated stretching, and has strong competitiveness in terms of stretching ability and gas barrier performance. The assembly strategy based on hydrogen bonding could be expanded in the construction of barrier materials used in flexible electronics, sensors and packing of devices.

Supporting Information Available:DipCoater image, Schematic diagram of tensile film of tensile testing machine and OTR measurement. This material is available free of charge via the Internet at XXX.

Acknowledgements. This work was supported by the National Natural Science Foundation of China (21671015, 21521005) and the Fundamental Research Funds for the Central Universities (XK1802-6, BHYC1702B, and XK1803-05).

\section{References}

(1) Xu, X.; Wang, L.; Wang, J.; Yin, Q.; Dong, S..; Han, J.; Wei, M., Hydroxide-ion-conductive gas barrier films based on layered double hydroxide/polysulfone multilayers. Chem. Comm. 2018;54:7778-7781.

(2) Di Maio, L.; Marra, F.; Bedane, T. F.; Incarnato, L.; Saguy, S., Oxygen transfer in co-extruded multilayer active films for food packaging. AlChE J. 2017;63: 5215-5221.

(3) Yang, C.; Cussler, E. L., Oxygen barriers that use free radical chemistry. AlChE J. 2001; 47: 2725-2732.

(4) Adel, P.; Moran, E.; Efrat, E.; Hadar, N. N.; Albert, R.; Israel, B., A novel hybrid solid dispersion film coat as a moisture barrier for pharmaceutical applications. J. Drug Delivery Sci. Technol.2017;40:105-115

(5) Dou, Y.; Pan, T.; Xu, S.; Yan, H.; Han, J.; Wei, M.; Evans, D. G.; Duan, X., Transparent, ultrahighgas-barrier films with a brick-mortar-sand structure.Angew. Chem. Int. Ed. 2015;54:9673-9678.

(6) Seethamraju, S.; Ramamurthy, P. C.; Madras, G., Performance of an ionomer blend-nanocomposite as an effective gas barrier material for organic devices. RSC Adv. 2014;4:11176-11187.

(7) Lee, M. E.; Jin, H. J., Nanocomposite films of poly(vinyl alcohol)-grafted graphene oxide/poly(vinyl alcohol) for gas barrier film applications. J. Nanosci. Nanotechnol. 2015;15:8348-8352.

(8) Gaume, J.; Taviot-Gueho, C.; Cros, S.; Rivaton, A.; Thérias, S.; Gardette, J. L., Optimization of PVA clay nanocomposite for ultra-barrier multilayer encapsulation of organic solar cells.Sol. Energy Mater. Sol. Cells 2012;99:240-249.

(9) Layek, R. K.; Das, A. K.; Min, U. P.; Kim, N. H.; Lee, J. H., Layer-structured graphene oxide/polyvinyl alcohol nanocomposites: dramatic enhancement of hydrogen gas barrier properties. J. Mater. Chem. A 2014;2:12158-12161.

(10) Zhang, J.; Lei, W.; Schutz, J.; Liu, D.; Tang, B.; Wang, C. H.; Wang, X., Improving the gas barrier, mechanical and thermal properties of poly(vinyl alcohol) with molybdenum disulfide nanosheets. J. Polym. Sci., Part B: Polym. Phys. 2019;57:406-414.

(11) Ham, M.; Kim, J. C.; Chang, J. H., Characterization of poly(vinyl alcohol) nanocomposite films with various clays. Polym. Korea2013; 37:225-231.

(12) Inagaki, N.; Tasaka, S.; Nakajima, T., Preparation of oxygen gas barrier polypropylene films by deposition of $\mathrm{SiO} x$ films plasma-polymerized from mixture of tetramethoxysilane and oxygen.J. Appl. Polym. Sci. 2000;78:2389-2397.

(13) Dou, Y.; Zhou, A.; Pan, T.; Han, J.; Wei, M.; Evans, D. G.; Duan, X., Humidity-triggered self-healing films with excellent oxygen barrier performance. Chem. Comm. 2014;50:7136-7138. 
(14) Wang, L.; Dou, Y.; Wang, J.; Han, J.; Liu, L.; Wei, M., Layer-by-layer assembly of layered double hydroxide/rubber multilayer films with excellent gas barrier property. Compos. Part A Appl. Sci. Manuf. 2017;102:314-321.

(15) Pan, T.; Xu, S.; Dou, Y.; Liu, X.; Li, Z..; Han, J.; Yan, H.; Wei, M., Remarkable oxygen barrier films based on layered double hydroxide/chitosan hierarchical structure. J. Mater. Chem. A2015:3;12350-12356.

(16) Cui, Y.; Kundalwal, S. I.; Kumar, S., Gas barrier performance of graphene/polymer nanocomposites. Carbon 2015;98:313-333.

(17) Petersen, H.; Jakubowicz, I.; Enebro, J.; Yarahmadi, N., Development of nanocomposites based on organically modified montmorillonite and plasticized PVC with improved barrier properties.J. Appl. Polym. Sci. 2015;133:189-196.

(18) Wang, P.; Wang, H.; Liu, J.; Wang, P.; Jiang, S.; Li, X.; Jiang, S., Montmorillonite@chitosan-poly (ethylene oxide) nanofibrous membrane enhancing poly (vinyl alcohol-co-ethylene) composite film. Carbohydr. Polym. 2018;181:885-892.

(19) Cao, P. F.; Li, B.; Hong, T.; Xing, K.; Voylov, D. N.; Cheng, S.; Yin, P.; Kisliuk, A. M.; Mahurin, S. M.; Sokolov, A. P., A robust and elastic polymer membrane with tunable properties for gas separation. ACS Appl. Mater. Interfaces 2017;9:26483-26491.

(20) Priolo, M. A.; Gamboa, D.; Grunlan, J. C., Transparent clay-polymer nano brick wall assemblies with tailorable oxygen barrier. ACS Appl. Mater. Interfaces 2010;2:312-320.

(21) Hagen, D. A.; Lauren, S.; Grunlan, J. C., Controlling effective aspect ratio and packing of clay with ph for improved gas barrier in nanobrick wall thin films. ACS Appl. Mater. Interfaces2014;6:22914-22919.

(22) Song, Y.; Gerringer, J.; Shuang, Q.; Grunlan, J. C., High oxygen barrier thin film from aqueous polymer/vlay slurry. Ind. Eng. Chem. Res. 2018;57:6904-6909.

(23) Zhai, X.; Li, Z.; Zhang, J.; Shi, J.; Zou, X.; Huang, X.; Zhang, D.; Sun, Y.; Yang, Z.; Holmes, M.; Gong, Y.; Povey, M., Natural biomaterial-based edible and ph-sensitive films combined with electrochemical writing for intelligent food packaging. J. Agric. Food. Chem. 2018;66,12836-12846.

(24) Dou, Y.; Xu, S.; Liu, X.; Han, J.; Yan, H.; Wei, M.; Evans, D. G.; Duan, X., Transparent, flexible films based on layered double hydroxide/cellulose acetate with excellent oxygen barrier property. Adv. Funct. Mater. 2014;24:514-521.

(25) Holder, K. M.; Spears, B. R.; Huff, M. E.; Priolo, M. A.; Harth, E.; Grunlan, J. C., Stretchable gas barrier achieved with partially hydrogen-bonded multilayer nanocoating. Macromol. Rapid Commun.2014;35:960-964.

(26) Xiang, F.; Ward, S. M.; Givens, T. M.; Grunlan, J. C., Super stretchy polymer multilayer thin film with high gas barrier. ACS Macro Lett. 2014;3:1055-1058.

(27) Xiang, F.; Givens, T. M.; Ward, S. M.; Grunlan, J. C., Elastomeric polymer multilayer thin film with sustainable gas barrier at high strain. ACS Appl. Mater. Interfaces 2015;7:16148-16151.

(28) Shuang, Q.; Song, Y.; Floto, M.; Grunlan, J. C., Combined high stretchability and gas barrier in hydrogen-bonded multilayer nanobrick wall thin films. ACS Appl. Mater. Interfaces 2017;9:7903-7907.

(29) Priolo, M. A.; Gamboa, D.; Holder, K. M.; Grunlan, J. C., Super gas barrier of transparent polymer-clay multilayer ultrathin films. Nano Lett. 2010;10:4970-4974.

(30) Zhao, M. Q.; Zhang, Q.; Huang, J. Q.; Wei, F., Hierarchical nanocomposites derived from nanocarbons and layered double hydroxides-properties, synthesis, and applications. Adv. Funct. Mater. 2012;22:675-694.

(31) Guo, X.; Zhang, F.; Evans, D. G.; Duan, X., Layered double hydroxide films: synthesis, properties and applications. Chem. Commun. 2010;46:5197-5210. 
(32) He, S.; An, Z.; Wei, M.; Evans, D. G.; Duan, X., Layered double hydroxide-based catalysts: nanostructure design and catalytic performance. Chem.Commun. 2013;44:5912-5920.

(33) Zi, G.; Atherton, J. J.; Zhi, P. X., Hierarchical layered double hydroxide nanocomposites: structure, synthesis and applications. Chem. Comm. 2015;51:3024-3036.

(34) Wang, Q.; O'Hare, D., Recent advances in the synthesis and application of layered double hydroxide (LDH) nanosheets. Chem. Rev. 2012;112:4124-4155.

(35) Dong, S.; Jia, Y.; Xu, X.; Luo, J.; Han, J., Crystallization and properties of poly(ethylene terephthalate)/layered double hydroxide nanocomposites. J. Colloid Interface Sci. 2018;539:54-64.

(36) Wang, J.; Pan, T.; Zhang, J.; Xu, X.; Yin, Q.; Han, J.; Wei, M., Hybrid films with excellent oxygen and water vapor barrier properties as efficient anticorrosive coatings. RSC Adv. 2018;8:21651-21657.

(37) Wang, J.; Xu, X.; Zhang, J.; Chen, M.; Dong. S.; Han, J.; Wei, M., Moisture-permeable, humidity-enhanced gas barrier films based on organic/inorganic multilayers. ACS Appl. Mater. Interfaces2018;33:28130-28138.

(38) Stockton, W. B.; Rubner, M. F., Molecular-level processing of conjugated polymers.4.layer-by-layer manipulation of polyaniline via hydrogen-bonding interactions. Macromolecules 1997;30:2717-2725.

(39) Yang, S. Y.; Rubner, M. F., Micropatterning of polymer thin films with ph-sensitive and cross-linkable hydrogen-bonded polyelectrolyte multilayers. J. Am. Chem. Soc. 2002;124:2100-2101.

(40) Cho, C.; Xiang, F.; Wallace, K. L.; Grunlan, J. C., Combined ionic and hydrogen bonding in polymer multilayer thin film for high gas barrier and stretchiness. Macromolecules 2015;48:5723-5729.

(41) Ji, N. M.; Jun, O. M.; Ho, C. J.; Yu, J. C.; Woo-Jae, K.; Juhyun, P.; Chang, Y. W.; Yoo, P. J., Layer-by-layer assembled multilayers of charged polyurethane and graphene oxide platelets for flexible and stretchable gas barrier films. Soft Matter. 2018;35:960-964.
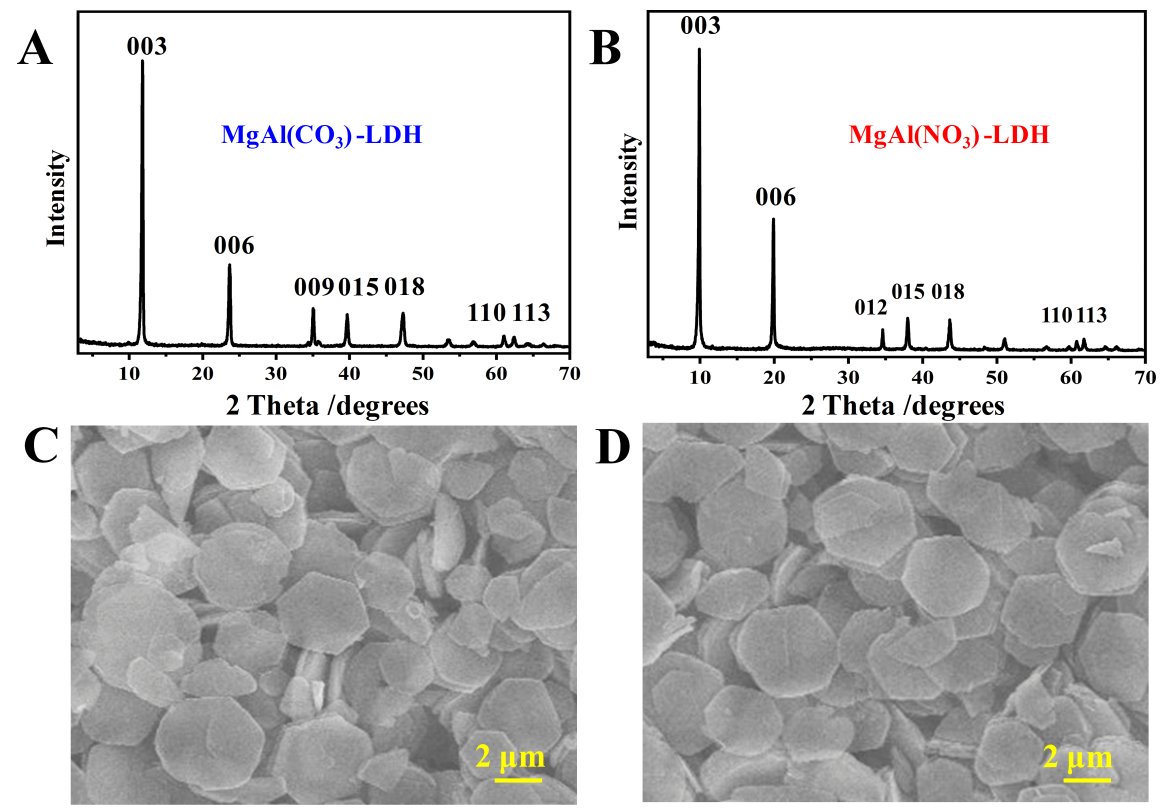

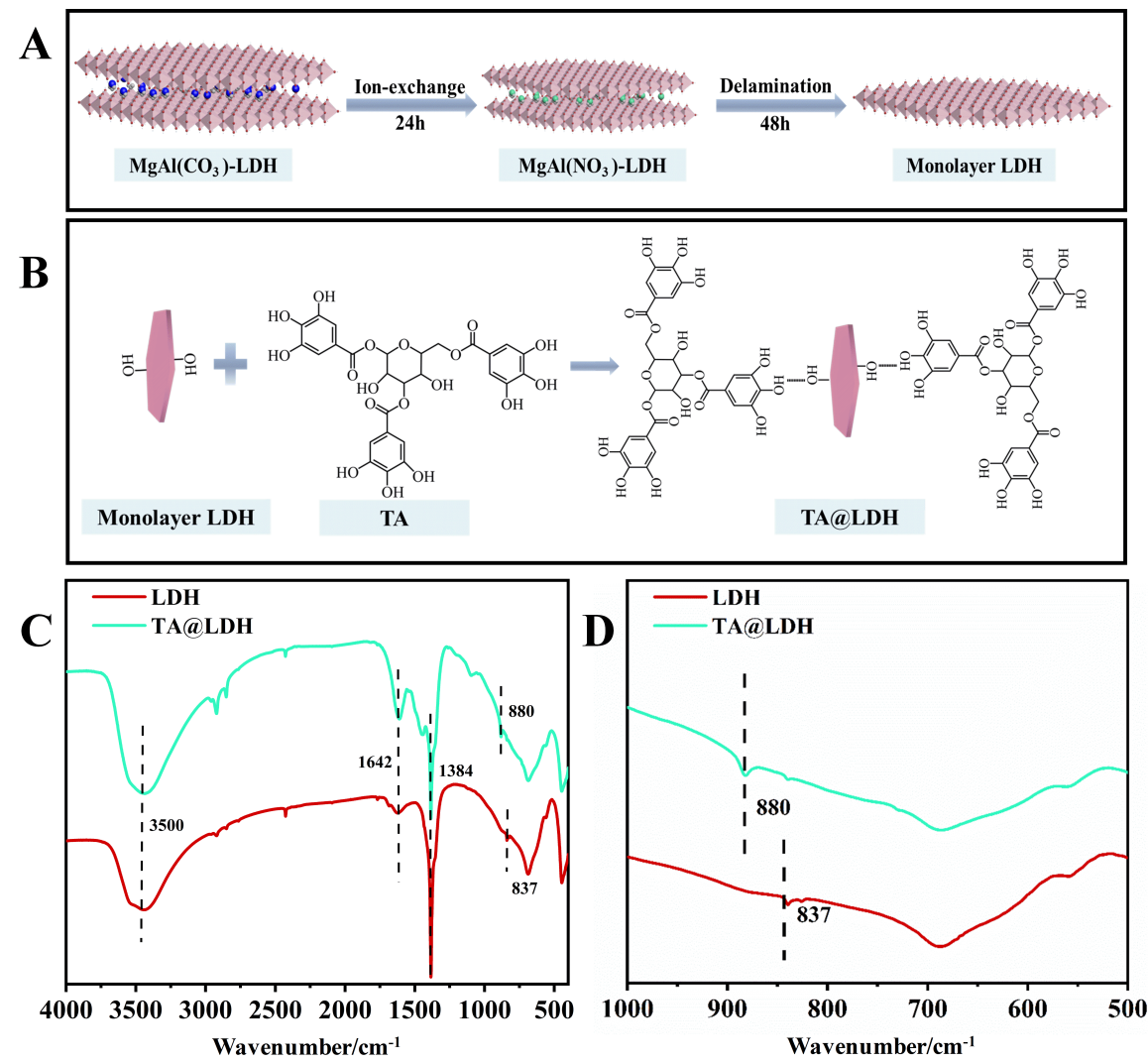

A
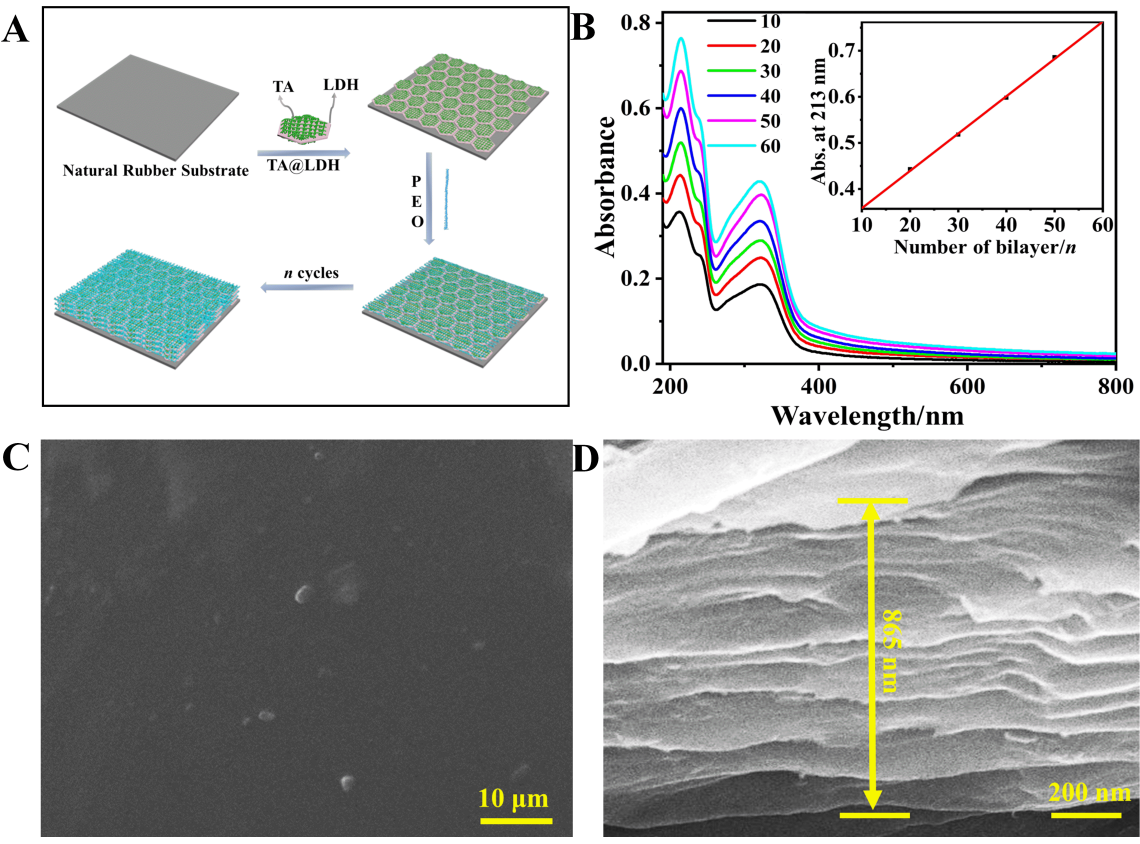

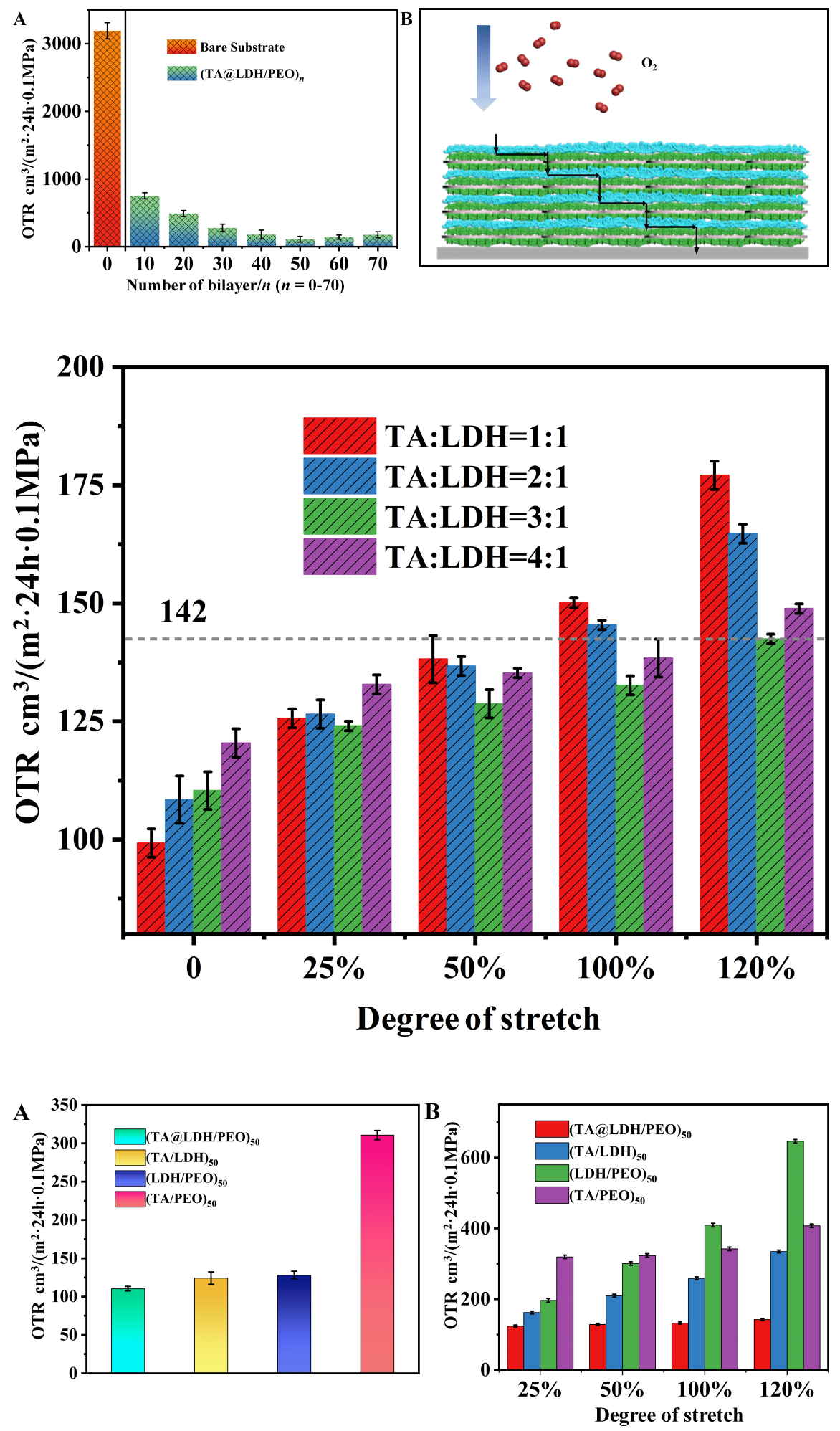

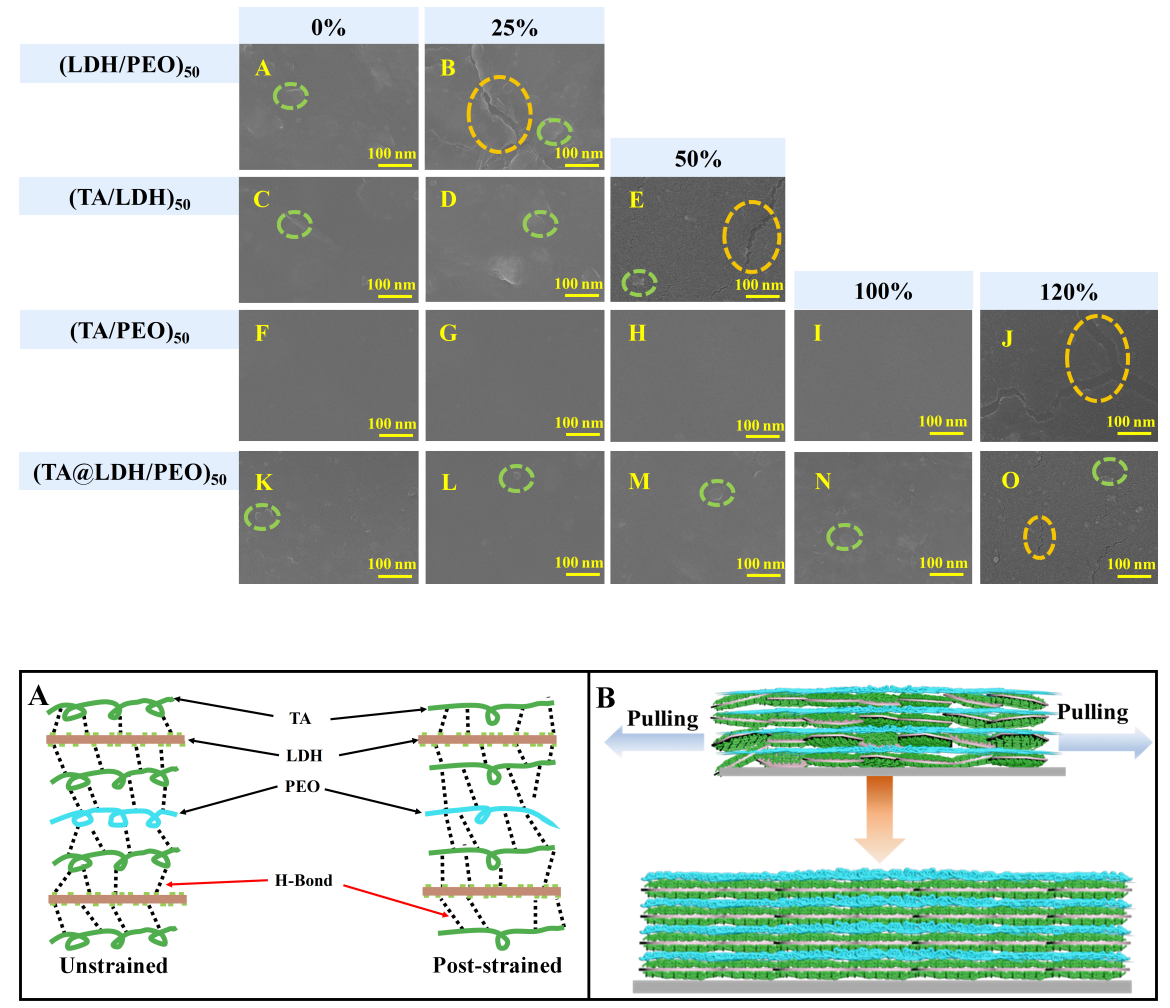